PROCEEDINGS OF THE

AMERICAN MATHEMATICAL SOCIETY

Volume 138, Number 3, March 2010, Pages 881-889

S 0002-9939(09)10183-1

Article electronically published on October 28, 2009

\title{
AN ASYMPTOTIC MEAN VALUE CHARACTERIZATION FOR $p$-HARMONIC FUNCTIONS
}

\author{
JUAN J. MANFREDI, MIKKO PARVIAINEN, AND JULIO D. ROSSI
}

(Communicated by Matthew J. Gursky)

To the memory of our friend and colleague Fuensanta Andreu

\begin{abstract}
We characterize $p$-harmonic functions in terms of an asymptotic mean value property. A $p$-harmonic function $u$ is a viscosity solution to $\Delta_{p} u=$ $\operatorname{div}\left(|\nabla u|^{p-2} \nabla u\right)=0$ with $1<p \leq \infty$ in a domain $\Omega$ if and only if the expansion

$$
u(x)=\frac{\alpha}{2}\left\{\frac{\max }{B_{\varepsilon}(x)} u+\frac{\min }{B_{\varepsilon}(x)} u\right\}+\frac{\beta}{\left|B_{\varepsilon}(x)\right|} \int_{B_{\varepsilon}(x)} u d y+o\left(\varepsilon^{2}\right)
$$

holds as $\varepsilon \rightarrow 0$ for $x \in \Omega$ in a weak sense, which we call the viscosity sense. Here the coefficients $\alpha, \beta$ are determined by $\alpha+\beta=1$ and $\alpha / \beta=(p-2) /(N+2)$.
\end{abstract}

\section{InTRODUCTION AND STATEMENT OF THE MAIN RESUlt}

A well known fact that one can find in any elementary PDE textbook states that $u$ is harmonic in a domain $\Omega \subset \mathbb{R}^{N}$ (that is, $u$ satisfies $\Delta u=0$ in $\Omega$ ) if and only if it satisfies the mean value property

$$
u(x)=\frac{1}{\left|B_{\varepsilon}(x)\right|} \int_{B_{\varepsilon}(x)} u(y) d y,
$$

whenever $B_{\varepsilon}(x) \subset \Omega$. In fact, we can relax this condition by requiring that it holds asymptotically:

$$
u(x)=\frac{1}{\left|B_{\varepsilon}(x)\right|} \int_{B_{\varepsilon}(x)} u(y) d y+o\left(\varepsilon^{2}\right),
$$

as $\varepsilon \rightarrow 0$. This follows easily for $C^{2}$ functions by using the Taylor expansion and for continuous functions by using the theory of viscosity solutions. Interestingly, a weak asymptotic mean value formula holds in some nonlinear cases as well. Our goal in this paper is to characterize $p$-harmonic functions, $1<p \leq \infty$, by means of this type of asymptotic mean value properties.

We begin by stating what we mean by weak asymptotic expansions and why it is reasonable to say that our asymptotic expansions hold in "a viscosity sense". As

Received by the editors January 9, 2009.

2010 Mathematics Subject Classification. Primary 35J92, 35J60, 35J70.

Key words and phrases. p-Laplacian, infinity Laplacian, mean value property, viscosity solutions.

The second author was supported by the Emil Aaltonen Foundation, the Fulbright Center, and the Magnus Ehrnrooth Foundation.

The third author was partially supported by project MTM2004-02223, MEC, Spain; by UBA X066; and by CONICET, Argentina. 
is the case in the theory of viscosity solutions, we test the expansions of a function $u$ against test functions $\phi$ that touch $u$ from below or above at a particular point.

Select $\alpha$ and $\beta$ determined by the conditions $\alpha+\beta=1$ and $\alpha / \beta=(p-2) /(N+2)$. That is, we have

$$
\alpha=\frac{p-2}{p+N} \quad \text { and } \quad \beta=\frac{2+N}{p+N} .
$$

Observe that if $p=2$ above, then $\alpha=0$ and $\beta=1$, and if $p=\infty$, then $\alpha=1$ and $\beta=0$.

We follow the usual convention to denote the mean value of a function:

$$
f_{B} f(y) d y=\frac{1}{|B|} \int_{B} f(y) d y
$$

Definition 1. A continuous function $u$ satisfies

$$
u(x)=\frac{\alpha}{2}\left\{\frac{\max }{B_{\varepsilon}(x)} u+\frac{\min }{B_{\varepsilon}(x)} u\right\}+\beta f_{B_{\varepsilon}(x)} u(y) d y+o\left(\varepsilon^{2}\right), \quad \text { as } \varepsilon \rightarrow 0,
$$

in the viscosity sense if

(1) for every $\phi \in C^{2}$ such that $u-\phi$ has a strict minimum at the point $x \in \bar{\Omega}$ with $u(x)=\phi(x)$, we have

$$
0 \geq-\phi(x)+\frac{\alpha}{2}\left\{\frac{\max }{B_{\varepsilon}(x)} \phi+\frac{\min }{B_{\varepsilon}(x)} \phi\right\}+\beta f_{B_{\varepsilon}(x)} \phi(y) d y+o\left(\varepsilon^{2}\right)
$$

(2) for every $\phi \in C^{2}$ such that $u-\phi$ has a strict maximum at the point $x \in \bar{\Omega}$ with $u(x)=\phi(x)$, we have

$$
0 \leq-\phi(x)+\frac{\alpha}{2}\left\{\frac{\max }{B_{\varepsilon}(x)} \phi+\frac{\min }{B_{\varepsilon}(x)} \phi\right\}+\beta f_{B_{\varepsilon}(x)} \phi(y) d y+o\left(\varepsilon^{2}\right) .
$$

Observe that a $C^{2}$-function $u$ satisfies (1.2) in the classical sense if and only if it satisfies it in the viscosity sense. However, the viscosity sense is actually weaker than the classical sense for non- $C^{2}$-functions as the following example, cf. [10], shows.

Example. Set $p=\infty$ and consider Aronsson's function

$$
u(x, y)=|x|^{4 / 3}-|y|^{4 / 3}
$$

near the point $(x, y)=(1,0)$. Aronsson's function is $\infty$-harmonic in the viscosity sense but it is not of class $C^{2}$; see Aronsson [1, 2]. It will follow from Theorem 2 below that $u$ satisfies

$$
u(x)=\frac{1}{2}\left\{\frac{\max }{B_{\varepsilon}(x)} u+\frac{\min }{B_{\varepsilon}(x)} u\right\}+o\left(\varepsilon^{2}\right) \quad \text { as } \varepsilon \rightarrow 0,
$$

in the viscosity sense of Definition 1. However, let us verify that the expansion does not hold in the classical sense.

Clearly, we have

$$
\frac{\max }{B_{\varepsilon}(1,0)} u=u(1+\varepsilon, 0)=(1+\varepsilon)^{4 / 3} .
$$


To find the minimum, we set $x=\varepsilon \cos (\theta), y=\varepsilon \sin (\theta)$ and solve the equation

$$
\begin{aligned}
\frac{d}{d \theta} u(1+\varepsilon \cos (\theta), \varepsilon \sin (\theta)) & =-\frac{4}{3}(1+\varepsilon \cos (\theta))^{1 / 3} \varepsilon \sin (\theta)-\frac{4}{3}(\varepsilon \sin (\theta))^{1 / 3} \varepsilon \cos (\theta) \\
& =0 .
\end{aligned}
$$

By symmetry, we can focus our attention on the solution

$$
\theta_{\varepsilon}=\arccos \left(\frac{\varepsilon-\sqrt{4+\varepsilon^{2}}}{2}\right) \text {. }
$$

Hence, we obtain

$$
\begin{aligned}
& \frac{\min }{B_{\varepsilon}(1,0)} u=u\left(1+\varepsilon \cos \left(\theta_{\varepsilon}\right), \varepsilon \sin \left(\theta_{\varepsilon}\right)\right) \\
& \quad=\left(1+\frac{1}{2} \varepsilon\left(\varepsilon-\sqrt{4+\varepsilon^{2}}\right)\right)^{4 / 3}-\left(\varepsilon \sqrt{1-\frac{1}{4}\left(\varepsilon-\sqrt{4+\varepsilon^{2}}\right)^{2}}\right)^{4 / 3} .
\end{aligned}
$$

We are ready to compute

$$
\lim _{\varepsilon \rightarrow 0+} \frac{\frac{1}{2}\left\{\frac{\max }{B_{\varepsilon}(1,0)} u+\frac{\min }{B_{\varepsilon}(1,0)} u\right\}-u(1,0)}{\varepsilon^{2}}=\frac{1}{18} .
$$

But if an asymptotic expansion held in the classical sense, this limit would have to be zero.

The following theorem states our main result and provides a characterization to the $p$-harmonic functions.

Theorem 2. Let $1<p \leq \infty$ and let $u$ be a continuous function in a domain $\Omega \subset \mathbb{R}^{N}$. The asymptotic expansion

$$
u(x)=\frac{\alpha}{2}\left\{\frac{\max }{B_{\varepsilon}(x)} u+\frac{\min }{B_{\varepsilon}(x)} u\right\}+\beta f_{B_{\varepsilon}(x)} u(y) d y+o\left(\varepsilon^{2}\right), \quad \text { as } \varepsilon \rightarrow 0,
$$

holds for all $x \in \Omega$ in the viscosity sense if and only if

$$
\Delta_{p} u(x)=0
$$

in the viscosity sense. Here $\alpha$ and $\beta$ are determined by (1.1).

We use the notation $\operatorname{div}\left(|\nabla u|^{p-2} \nabla u\right)=\Delta_{p} u$ for the regular $p$-Laplacian and $\Delta_{\infty} u=|\nabla u|^{-2}\left\langle D^{2} u \nabla u, \nabla u\right\rangle$ for the 1-homogeneous infinity Laplacian. The infinity Laplacian appears naturally when one considers limits as $p \rightarrow \infty$ of $p$-harmonic functions in the viscosity sense and has applications to best Lipschitz extensions, image processing and mass transport problems; see [2], 3], 4], 7], 8], 9].

We observe that the notions of a viscosity solution and a Sobolev weak solution for the $p$-Laplace equation agree for $1<p<\infty$; see Juutinen-LindqvistManfredi [11. Therefore, Theorem[2] characterizes weak solutions when $1<p<\infty$.

Finally, we note that Wang [14] has also used Taylor series to give sufficient conditions for $p$-subharmonicity in terms of asymptotic mean values of $(u(x)-$ $u(0))^{p}$. 


\section{Proof of Theorem 2}

To gain some intuition as to why such an asymptotic mean value formula might be true, let us formally expand the $p$-Laplacian as follows:

$$
\Delta_{p} u=(p-2)|\nabla u|^{p-4}\left\langle D^{2} u \nabla u, \nabla u\right\rangle+|\nabla u|^{p-2} \Delta u .
$$

This formal expansion was used by Peres and Sheffield in 13 (see also Peres et al. [12]) to find $p$-harmonic functions as limits of values of Tug-of-War games.

Suppose that $u$ is a smooth function with $\nabla u \neq 0$. We see from (2.1) that $u$ is a solution to $\Delta_{p} u=0$ if and only if

$$
(p-2) \Delta_{\infty} u+\Delta u=0 .
$$

It follows from the classical Taylor expansion that

$$
u(x)-f_{B_{\varepsilon}(x)} u d y=-\varepsilon^{2} \Delta u(x) \frac{1}{2 N} f_{B(0,1)}|z|^{2} d z+o\left(\varepsilon^{2}\right)
$$

and

$$
\begin{aligned}
u(x) & -\frac{1}{2}\left\{\frac{\max }{B_{\varepsilon}(x)} u+\frac{\min }{B_{\varepsilon}(x)} u\right\} \\
& \approx u(x)-\frac{1}{2}\left\{u\left(x+\varepsilon \frac{\nabla u(x)}{|\nabla u(x)|}\right)+u\left(x-\varepsilon \frac{\nabla u(x)}{|\nabla u(x)|}\right)\right\} \\
& =-\frac{\varepsilon^{2}}{2} \Delta_{\infty} u(x)+o\left(\varepsilon^{2}\right) .
\end{aligned}
$$

The volume of the unit ball in $\mathbb{R}^{N}$ will be denoted by $\omega_{N}$ and the $(N-1)$ dimensional area of the unit sphere will be denoted by $\sigma_{N-1}$. Observe that since $\sigma_{N-1} / \omega_{N}=N$ we have

$$
\frac{1}{N} f_{B(0,1)}|z|^{2} d z=\frac{1}{N+2} .
$$

Multiply (2.3) and (2.4) by suitable constants and add up the formulas so that we have the operator from (2.2) on the right hand side. This process gives us the choices of the constants $\alpha$ and $\beta$ in (1.1) needed to obtain the asymptotic expansion of Theorem 2 ,

The main idea of the proof of Theorem 2 is just to work in the viscosity setting and use the expansions (2.3) and (2.4). The derivation of (2.4) also needs some care. We start by recalling the viscosity characterization of $p$-harmonic functions for $p<\infty$; see [11].

Definition 3. For $1<p<\infty$ consider the equation $-\operatorname{div}\left(|\nabla u|^{p-2} \nabla u\right)=0$.

(1) A lower semi-continuous function $u$ is a viscosity supersolution if for every $\phi \in C^{2}$ such that $u-\phi$ has a strict minimum at the point $x \in \Omega$ with $\nabla \phi(x) \neq 0$ we have

$$
-(p-2) \Delta_{\infty} \phi(x)-\Delta \phi(x) \geq 0 .
$$

(2) An upper semi-continuous function $u$ is a subsolution if for every $\phi \in C^{2}$ such that $u-\phi$ has a strict maximum at the point $x \in \Omega$ with $\nabla \phi(x) \neq 0$ we have

$$
-(p-2) \Delta_{\infty} \phi(x)-\Delta \phi(x) \leq 0 .
$$


(3) Finally, $u$ is a viscosity solution if it is both a supersolution and a subsolution.

For the case $p=\infty$ we must restrict the class of test functions as in 12. Let $S(x)$ denote the class of $C^{2}$ functions $\phi$ such that either $\nabla \phi(x) \neq 0$ or $\nabla \phi(x)=0$ and the limit

$$
\lim _{y \rightarrow x} \frac{2(\phi(y)-\phi(x))}{|y-x|^{2}}=\Delta_{\infty} \phi(x)
$$

exists.

Definition 4. Consider the equation $-\Delta_{\infty} u=0$.

(1) A lower semi-continuous function $u$ is a viscosity supersolution if for every $\phi \in S(x)$ such that $u-\phi$ has a strict minimum at the point $x \in \Omega$ we have

$$
-\Delta_{\infty} \phi(x) \geq 0 .
$$

(2) An upper semi-continuous function $u$ is a subsolution if for every $\phi \in S(x)$ such that $u-\phi$ has a strict maximum at the point $x \in \Omega$ we have

$$
-\Delta_{\infty} \phi(x) \leq 0 .
$$

(3) Finally, $u$ is a viscosity solution if it is both a supersolution and a subsolution.

Proof of Theorem 2. We first consider asymptotic expansions for smooth functions that involve the infinity Laplacian $(p=\infty)$ and the regular Laplacian $(p=2)$.

Choose a point $x \in \Omega$ and a $C^{2}$-function $\phi$ defined in a neighborhood of $x$. Let $x_{1}^{\varepsilon}$ and $x_{2}^{\varepsilon}$ be the points at which $\phi$ attains its minimum and maximum in $\overline{B_{\varepsilon}(x)}$ respectively, that is,

$$
\phi\left(x_{1}^{\varepsilon}\right)=\min _{y \in \overline{B_{\varepsilon}(x)}} \phi(y) \quad \text { and } \quad \phi\left(x_{2}^{\varepsilon}\right)=\max _{y \in \overline{B_{\varepsilon}(x)}} \phi(y) .
$$

Next, we use some ideas from [5]. Consider the Taylor expansion of the second order of $\phi$ :

$$
\phi(y)=\phi(x)+\nabla \phi(x) \cdot(y-x)+\frac{1}{2}\left\langle D^{2} \phi(x)(y-x),(y-x)\right\rangle+o\left(|y-x|^{2}\right)
$$

as $|y-x| \rightarrow 0$. Evaluating this Taylor expansion of $\phi$ at the point $x$ with $y=x_{1}^{\varepsilon}$ and $y=2 x-x_{1}^{\varepsilon}=\tilde{x}_{1}^{\varepsilon}$, we get

$$
\phi\left(x_{1}^{\varepsilon}\right)=\phi(x)+\nabla \phi(x)\left(x_{1}^{\varepsilon}-x\right)+\frac{1}{2}\left\langle D^{2} \phi(x)\left(x_{1}^{\varepsilon}-x\right),\left(x_{1}^{\varepsilon}-x\right)\right\rangle+o\left(\varepsilon^{2}\right)
$$

and

$$
\phi\left(\tilde{x}_{1}^{\varepsilon}\right)=\phi(x)-\nabla \phi(x)\left(x_{1}^{\varepsilon}-x\right)+\frac{1}{2}\left\langle D^{2} \phi(x)\left(x_{1}^{\varepsilon}-x\right),\left(x_{1}^{\varepsilon}-x\right)\right\rangle+o\left(\varepsilon^{2}\right)
$$

as $\varepsilon \rightarrow 0$. Adding the expressions, we obtain

$$
\phi\left(\tilde{x}_{1}^{\varepsilon}\right)+\phi\left(x_{1}^{\varepsilon}\right)-2 \phi(x)=\left\langle D^{2} \phi(x)\left(x_{1}^{\varepsilon}-x\right),\left(x_{1}^{\varepsilon}-x\right)\right\rangle+o\left(\varepsilon^{2}\right) .
$$

Since $x_{1}^{\varepsilon}$ is the point where the minimum of $\phi$ is attained, it follows that

$$
\phi\left(\tilde{x}_{1}^{\varepsilon}\right)+\phi\left(x_{1}^{\varepsilon}\right)-2 \phi(x) \leq \max _{y \in \overline{B_{\varepsilon}(x)}} \phi(y)+\min _{y \in \overline{B_{\varepsilon}(x)}} \phi(y)-2 \phi(x),
$$


and thus

$$
\begin{aligned}
\frac{1}{2}\left\{\max _{y \in \overline{B_{\varepsilon}(x)}} \phi(y)\right. & \left.+\min _{y \in \overline{B_{\varepsilon}(x)}} \phi(y)\right\}-\phi(x) \\
& \geq \frac{1}{2}\left\langle D^{2} \phi(x)\left(x_{1}^{\varepsilon}-x\right),\left(x_{1}^{\varepsilon}-x\right)\right\rangle+o\left(\varepsilon^{2}\right) .
\end{aligned}
$$

Repeating the same process at the point $x_{2}^{\varepsilon}$ we get instead

$$
\begin{aligned}
\frac{1}{2}\left\{\max _{y \in \overline{B_{\varepsilon}(x)}} \phi(y)\right. & \left.+\min _{y \in \overline{B_{\varepsilon}(x)}} \phi(y)\right\}-\phi(x) \\
& \leq \frac{1}{2}\left\langle D^{2} \phi(x)\left(x_{2}^{\varepsilon}-x\right),\left(x_{2}^{\varepsilon}-x\right)\right\rangle+o\left(\varepsilon^{2}\right) .
\end{aligned}
$$

Next we derive a counterpart for the expansion with the usual Laplacian $(p=2)$. Averaging both sides of the classical Taylor expansion of $\phi$ at $x$ we get

$$
f_{B_{\varepsilon}(x)} \phi(y) d y=\phi(x)+\sum_{i, j=1}^{N} \frac{\partial^{2} \phi}{\partial x_{i}^{2}}(x) f_{B_{\varepsilon}(0)} \frac{1}{2} z_{i} z_{j} d z+o\left(\varepsilon^{2}\right) .
$$

The values of the integrals in the sum above are zero when $i \neq j$. Using symmetry, we compute

$$
\begin{aligned}
& f_{B_{\varepsilon}(0)} z_{i}^{2} d z=\frac{1}{N} f_{B_{\varepsilon}(0)}|z|^{2} d z \\
& =\frac{1}{N \omega_{N} \varepsilon^{N}} \int_{0}^{\varepsilon} \int_{\partial B_{\rho}} \rho^{2} d S d \rho=\frac{\sigma_{N-1} \varepsilon^{2}}{N(N+2) \omega_{N}}=\frac{\varepsilon^{2}}{(N+2)},
\end{aligned}
$$

with the notation introduced after (2.4). We end up with

$$
f_{B_{\varepsilon}(x)} \phi(y) d y-\phi(x)=\frac{\varepsilon^{2}}{2(N+2)} \Delta \phi(x)+o\left(\varepsilon^{2}\right) .
$$

Assume for the moment that $p \geq 2$ so that $\alpha \geq 0$. Multiply (2.5) by $\alpha$ and (2.7) by $\beta$ and add. We arrive at the expansion valid for any smooth function $\phi$ :

$$
\begin{aligned}
& \frac{\alpha}{2}\left\{\max _{y \in \overline{B_{\varepsilon}(x)}} \phi(y)+\min _{y \in \overline{B_{\varepsilon}(x)}} \phi(y)\right\}+\beta f_{B_{\varepsilon}(x)} \phi(y) d y-\phi(x) \\
& \geq \frac{\beta \varepsilon^{2}}{2(N+2)}\left((p-2)\left\langle D^{2} \phi(x)\left(\frac{x_{1}^{\varepsilon}-x}{\varepsilon}\right),\left(\frac{x_{1}^{\varepsilon}-x}{\varepsilon}\right)\right\rangle+\Delta \phi(x)\right) \\
& \quad+o\left(\varepsilon^{2}\right) .
\end{aligned}
$$

We remark that $x_{1}^{\varepsilon} \in \partial B_{\varepsilon}(x)$ for $\varepsilon>0$ small enough whenever $\nabla \phi(x) \neq 0$. In fact, suppose, on the contrary, that there exists a subsequence $x_{1}^{\varepsilon_{j}} \in B_{\varepsilon_{j}}(x)$ of minimum points of $\phi$. Then $\nabla \phi\left(x_{1}^{\varepsilon_{j}}\right)=0$ and, since $x_{1}^{\varepsilon_{j}} \rightarrow x$ as $\varepsilon_{j} \rightarrow 0$, we have by continuity that $\nabla \phi(x)=0$. A simple argument based on Lagrange multipliers then shows that

$$
\lim _{\varepsilon \rightarrow 0} \frac{x_{1}^{\varepsilon}-x}{\varepsilon}=-\frac{\nabla \phi}{|\nabla \phi|}(x) .
$$

We are ready to prove that if the asymptotic mean value formula holds for $u$, then $u$ is a viscosity solution. Suppose that the function $u$ satisfies the asymptotic 
expansion in the viscosity sense according to Definition 11 Consider a smooth $\phi$ such that $u-\phi$ has a strict minimum at $x$ and $\phi \in S(x)$ if $p=\infty$. We obtain

$$
0 \geq-\phi(x)+\frac{\alpha}{2}\left\{\frac{\max }{B_{\varepsilon}(x)} \phi+\frac{\min }{B_{\varepsilon}(x)} \phi\right\}+\beta f_{B_{\varepsilon}(x)} \phi(y) d y+o\left(\varepsilon^{2}\right),
$$

and thus, by (2.8),

$$
0 \geq \frac{\beta \varepsilon^{2}}{2(N+2)}\left((p-2)\left\langle D^{2} \phi(x)\left(\frac{x_{1}^{\varepsilon}-x}{\varepsilon}\right),\left(\frac{x_{1}^{\varepsilon}-x}{\varepsilon}\right)\right\rangle+\Delta \phi(x)\right)+o\left(\varepsilon^{2}\right) .
$$

If $\nabla \phi(x) \neq 0$ we take limits as $\varepsilon \rightarrow 0$. Taking into consideration (2.9) we get

$$
0 \geq \frac{\beta}{2(N+2)}\left((p-2) \Delta_{\infty} \phi(x)+\Delta \phi(x)\right) .
$$

Suppose now that $p=\infty$ and that the limit

$$
\lim _{y \rightarrow x} \frac{\phi(y)-\phi(x)}{|y-x|^{2}}=L
$$

exists. We need to deduce that $L \leq 0$ from

$$
0 \geq \frac{1}{2}\left\{\frac{\max }{B_{\varepsilon}(x)} \phi+\frac{\min }{B_{\varepsilon}(x)} \phi\right\}-\phi(x) .
$$

Let us argue by contradiction. Suppose that $L>0$ and choose $\eta>0$ small enough so that $L-\eta>0$. Use the limit condition to obtain the inequalities

$$
(L-\eta)|x-y|^{2} \leq \phi(x)-\phi(y) \leq(L+\eta)|x-y|^{2},
$$

for small $|x-y|$. Therefore, we get

$$
\begin{aligned}
0 & \geq \frac{1}{2} \frac{\max }{B_{\varepsilon}(x)}(\phi-\phi(x))+\frac{1}{2} \frac{\min }{B_{\varepsilon}(x)}(\phi-\phi(x)) \\
& \geq \frac{1}{2} \frac{\max }{B_{\varepsilon}(x)}(\phi-\phi(x)) \\
& \geq\left(\frac{L-\eta}{2}\right) \varepsilon^{2},
\end{aligned}
$$

which is a contradiction. Thus, we have proved that $L \geq 0$.

To prove that $u$ is a viscosity subsolution, we first derive a reverse inequality to (2.8) by considering the maximum point of the test function, that is, using (2.6) and (2.7), and then choose a function $\phi$ that touches $u$ from above. We omit the details.

To prove the converse implication, assume that $u$ is a viscosity solution. In particular $u$ is a subsolution. Let $\phi$ be a smooth test function such that $u-\phi$ has a strict local maximum at $x \in \Omega$. If $p=\infty$, we also assume $\phi \in S(x)$. If $\nabla \phi(x) \neq 0$, we get

$$
-(p-2) \Delta_{\infty} \phi(x)-\Delta \phi(x) \leq 0 .
$$

The statement to be proven is

$$
\liminf _{\varepsilon \rightarrow 0+} \frac{1}{\varepsilon^{2}}\left(-\phi(x)+\frac{\alpha}{2}\left\{\frac{\max }{B_{\varepsilon}(x)} \phi+\frac{\min }{B_{\varepsilon}(x)} \phi\right\}+\beta f_{B_{\varepsilon}(x)} \phi(y) d y\right) \geq 0 .
$$


This again follows from (2.8). Indeed, divide (2.8) by $\varepsilon^{2}$, use (2.9), and deduce from (2.10) that the limit on the right hand side is bounded from below by zero.

For the case $p=\infty$ with $\nabla \phi(x)=0$ we assume the existence of the limit

$$
\lim _{y \rightarrow x} \frac{\phi(y)-\phi(x)}{|y-x|^{2}}=L \geq 0
$$

and observe that

$$
\liminf _{\varepsilon \rightarrow 0+} \frac{1}{\varepsilon^{2}}\left(-\phi(x)+\frac{1}{2}\left\{\frac{\max }{B_{\varepsilon}(x)} \phi+\frac{\min }{B_{\varepsilon}(x)} \phi\right\}\right) \geq 0 .
$$

The argument for the case of supersolutions is analogous.

Finally, we need to address the case $1<p<2$. Since $\alpha \leq 0$ we use (2.6) instead of (2.5) to get a version of (2.8) with $x_{2}^{\varepsilon}$ in place of $x_{1}^{\varepsilon}$. The argument then continues in the same way as before.

\section{ACKNOWLEDGMENT}

Part of this work was done during a visit of the third author to the University of Pittsburgh. He wants to thank them for the friendly and stimulating atmosphere found there.

\section{REFERENCES}

1. G. Aronsson, Extension of functions satisfying Lipschitz conditions. Ark. Mat., 6 (1967), 551-561. MR0217665 (36:754)

2. G. Aronsson, M.G. Crandall and P. Juutinen, A tour of the theory of absolutely minimizing functions. Bull. Amer. Math. Soc. (N.S.), 41 (2004), 439-505. MR2083637(2005k:35159)

3. E.N. Barron, L.C. Evans and R. Jensen, The infinity Laplacian, Aronsson's equation and their generalizations. Trans. Amer. Math. Soc., 360 (2008), 77-101. MR2341994

4. T. Bhattacharya, E. DiBenedetto and J. Manfredi, Limits as $p \rightarrow \infty$ of $\Delta_{p} u_{p}=f$ and related extremal problems. Rend. Sem. Mat. Univ. Politec. Torino, 1989, Special Issue (1991), 15-68. MR.1155453 (93a:35049)

5. F. Charro, J. Garcia Azorero and J. D. Rossi, A mixed problem for the infinity Laplacian via tug-of-war games. Calc. Var. Partial Differential Equations, 34 (2009), 307-320. MR2471139

6. M.G. Crandall, H. Ishii and P.-L. Lions, User's guide to viscosity solutions of second order partial differential equations. Bull. Amer. Math. Soc. (N.S.), 27 (1992), 1-67. MR.1118699 (92j:35050)

7. L.C. Evans and W. Gangbo, Differential equations methods for the Monge-Kantorovich mass transfer problem. Mem. Amer. Math. Soc., 137 (1999), no. 653. MR1464149 (99g:35132)

8. J. García-Azorero, J.J. Manfredi, I. Peral and J.D. Rossi, The Neumann problem for the $\infty$-Laplacian and the Monge-Kantorovich mass transfer problem. Nonlinear Analysis TM\&A, 66(2) (2007), 349-366. MR2279530 (2008f:35148)

9. R. Jensen, Uniqueness of Lipschitz extensions: Minimizing the sup norm of the gradient. Arch. Rational Mech. Anal., 123 (1993), 51-74. MR.1218686 (94g:35063)

10. E. Le Gruyer, On absolutely minimizing Lipschitz extensions and PDE $\Delta_{\infty}(u)=0$, NoDEA Nonlinear Differ. Equ. Appl., 14 (2007), 29-55. MR2346452(2008k:35159)

11. P. Juutinen, P. Lindqvist and J.J. Manfredi, On the equivalence of viscosity solutions and weak solutions for a quasi-linear elliptic equation, SIAM J. Math. Anal., 33 (2001), 699-717. MR $1871417(2002 \mathrm{~m}: 35051)$

12. Y. Peres, O. Schramm, S. Sheffield and D. Wilson, Tug-of-war and the infinity Laplacian. J. Amer. Math. Soc., 22 (2009), 167-210. MR2449057(2009h:91004) 
13. Y. Peres and S. Sheffield, Tug-of-war with noise: A game theoretic view of the p-Laplacian. Duke Math. J. 145(1) (2008), 91-120. MR2451291.

14. P. Wang, A formula for smooth $\infty$-harmonic functions, PanAmerican Mathematical Journal, 16 (2006), no. 1, 57-65. MR2186538

Department of Mathematics, University of Pittsburgh, Pittsburgh, Pennsylvania 15260

E-mail address: manfredi@pitt.edu

Institute of Mathematics, Helsinki University of Technology, P.O. Box 1100, 02015 TKK, Helsinki, Finland

E-mail address: Mikko.Parviainen@tkk.fi

imdea Matemáticas, C-IX, Universidad Autónoma de Madrid, 28049 Madrid, Spain

Current address: FCEyN UBA (1428), Buenos Aires, Argentina

E-mail address: jrossi@dm.uba.ar 